\title{
Increasing Spontaneous Retinal Activity before Eye Opening Accelerates the Development of Geniculate Receptive Fields
}

\author{
(Dachary W. Davis, ${ }^{1}$ Barbara Chapman, ${ }^{1,2}$ and ${ }^{-H}$ Hai-Jong Cheng ${ }^{1,2,3}$ \\ ${ }^{1}$ Center for Neuroscience, ${ }^{2}$ Department of Neurobiology, Physiology, and Behavior, and ${ }^{3}$ Department of Pathology and Laboratory Medicine, University of \\ California, Davis, California 95618
}

Visually evoked activity is necessary for the normal development of the visual system. However, little is known about the capacity for patterned spontaneous activity to drive the maturation of receptive fields before visual experience. Retinal waves provide instructive retinotopic information for the anatomical organization of the visual thalamus. To determine whether retinal waves also drive the maturation of functional responses, we increased the frequency of retinal waves pharmacologically in the ferret (Mustela putorius furo) during a period of retinogeniculate development before eye opening. The development of geniculate receptive fields after receiving these increased neural activities was measured using single-unit electrophysiology. We found that increased retinal waves accelerate the developmental reduction of geniculate receptive field sizes. This reduction is due to a decrease in receptive field center size rather than an increase in inhibitory surround strength. This work reveals an instructive role for patterned spontaneous activity in guiding the functional development of neural circuits.

Key words: electrophysiology; ferret; LGN; receptive field; retinal waves; spontaneous activity

Significance Statement

Patterned spontaneous neural activity that occurs during development is known to be necessary for the proper formation of neural circuits. However, it is unknown whether the spontaneous activity alone is sufficient to drive the maturation of the functional properties of neurons. Our work demonstrates for the first time an acceleration in the maturation of neural function as a consequence of driving patterned spontaneous activity during development. This work has implications for our understanding of how neural circuits can be modified actively to improve function prematurely or to recover from injury with guided interventions of patterned neural activity.

\section{Introduction}

Neural activity is essential for the modification of neural circuits. Enriching the sensory environment can accelerate development (Schwartz et al., 2011; Liu et al., 2012), improve recovery from injury (Baroncelli et al., 2012; Yu et al., 2014), and increase performance of learning and memory (Falkenberg et al., 1992). However, it is less clear how enrichment of spontaneous activity, especially during periods when environmentally evoked activity

Received April 9, 2015; revised Sept. 17, 2015; accepted Sept. 24, 2015.

Author contributions: Z.W.D., B.C., and H.-J.C. designed research; Z.W.D. performed research; Z.W.D. analyzed data; Z.W.D. and H.-J.C. wrote the paper.

This work was supported by the National Institutes of Health-National Eye Institute (Grants EY11369 and EY020743 to B.C. and H.J.-C. and Vision Training Grant EY015387 to Jack Werner). We thank Marty Usrey, Samuel Failor, Karl Murray, and Julie Luu for advice on the techniques used in this manuscript; Daniel Sperka for technical help; and Ana Marija Sola for help with data analysis.

Correspondence should be addressed to Hwai--Jong Cheng, Center for Neuroscience, University of California, 1544 Newton Ct., Davis, CA 95618. E-mail: hjcheng@ucdavis.edu.

DOI:10.1523/JNEUROSCI.1365-15.2015

Copyright $\odot 2015$ the authors $\quad 0270-6474 / 15 / 3514612-12 \$ 15.00 / 0$ is absent, influences the organization and maturation of the nervous system.

Patterned spontaneous activity instructs the development of neural circuits (Penn et al., 1998; Stellwagen and Shatz, 2002; Blankenship and Feller, 2010; Xu et al., 2011; Burbridge et al., 2014). Disruption or blockade results in failures in the development of the functional properties of neurons such as receptive field size, organization, and tuning (Archer et al., 1982; Stryker and Harris, 1986; Chapman and Gödecke, 2000; Huberman et al., 2006; Failor et al., 2015), but it remains an open question as to how driving spontaneous activity can positively influence the development of a neural circuit. Using the development of the visual system in the ferret (Mustela putorius furo) as a model for activity-dependent plasticity, we sought to investigate how driving postnatal spontaneous activity can promote the maturation of neural circuits.

In the visual system, gradients of axon guidance molecules (Huberman et al., 2005; Pfeiffenberger et al., 2005) and patterned spontaneous activity in the form of retinal waves (Meister et al., 1991) instruct the anatomical organization of retinal ganglion 
cell (RGC) projections in the thalamus and superior colliculus (Penn et al., 1998; Bansal et al., 2000; Stellwagen and Shatz, 2002; Furman et al., 2013) while visually evoked activity instructs the functional development and maturation of visual responses (Chen and Regehr, 2000; Tavazoie and Reid, 2000; Akerman et al., 2002; Hooks and Chen, 2006). The functional properties of relay cells in the dorsal lateral geniculate nucleus (dLGN) of the thalamus are immature at eye opening. During the course of visual experience, the number of individual RGCs connecting to a geniculate neuron decreases and the synaptic efficacy of the remaining inputs greatly increases (Chen and Regehr, 2000). This coincides with a developmental decrease in the size of geniculate receptive fields and an increase in the strength of visually evoked responses (Tavazoie and Reid, 2000). When animals are dark reared, some functional properties of RGCs and geniculate neurons fail to mature (Akerman et al., 2002; Tian and Copenhagen, 2003). However, the refinement of ganglion cell arbors can still occur after dark rearing, suggesting that spontaneous activity plays a critical role in this process (Hooks and Chen, 2006).

Due to the negative consequences of losses of activity on the development of the retina (Bisti et al., 1998; Kerschensteiner et al., 2009), we chose to assay the influence of spontaneous retinal activity on the development of geniculate receptive fields through a gain of activity model. Our hypothesis is that increasing patterned spontaneous retinal activity before visual experience will drive retinotopic refinement of RGC inputs to the dLGN and accelerate the refinement of geniculate receptive fields as made evident by a decrease in receptive field size. Using two different pharmacological approaches, we increased the frequency of patterned spontaneous activity (including spikes, bursts, and retinal waves) during a period of glutamatergic retinal waves before eye opening in the ferret.

To quantify the developmental consequence of this treatment, we measured the size of geniculate receptive fields after eye opening and examined the trajectory of developmental refinement as a consequence of normal visual experience. Ferrets that experienced increased spontaneous retinal activity had smaller geniculate receptive fields at eye opening compared with saline-treated controls. This treatment had no effect on the developmental refinement that occurs due to visual experience after eye opening or on the development of surround inhibition. From this, we conclude that driving patterned spontaneous activity can instruct the development of the functional properties of neural circuits.

\section{Materials and Methods}

Animals. Timed pregnant and neonatal litters of fitch-coat ferrets were obtained from Marshall Farms. All experimental procedures were performed in accordance with approved animal use protocols at the University of California-Davis. Ferrets were monitored for normal weight gain and growth throughout the course of the study (Chapman and Gödecke, 2000), as well as for postoperative distress from the experimental procedures.

Intraocular injections. Surgical and drug injection procedures were similar to those described previously (Huberman et al., 2002; Speer et al., 2010; Davis et al., 2015). Mixed-gender juvenile ferrets were anesthetized with inhalant isofluorane (Isothesia; Butler Schein), the eyelids were opened, and a small incision was made on the temporal side at the joining of the upper and lower eyelid. Binocular intravitreal injections of sterile saline, (RS)-a-cyclopropyl-4-phosphonophenylglycine (CPPG) (2 mM), or a combination of (1,2,5,6-tetrahydropyridin-4-yl)-methylphosphinic acid (TPMPA) $(2 \mathrm{mM})$, gabazine $(200 \mu \mathrm{M})$, and strychnine $(20 \mu \mathrm{M})$ [this combination is hereafter referred to as TGS] were administered daily for 10 consecutive days starting at postnatal day 15 (P15). This starting age was chosen to capture a period of time during glutamatergic retinal waves in the ferret after eye-specific segregation in the dLGN and preceding light-evoked activity in the retina. The end age of P25 was chosen to reduce the risk of damage to the eye from repeated daily injections and to prevent the persistent disruption of light-evoked activity after the retina becomes photoactive. LGN recordings began around the age of normal eye opening in the ferret (P30)

The high concentrations of our pharmacological agents were chosen to achieve intraocular concentrations of $500 \mu \mathrm{M}$ CPPG or $50 \mu \mathrm{M}$ TPMPA, 5 $\mu \mathrm{M}$ gabazine, and $500 \mathrm{~nm}$ strychnine after diffusion into the eye. Because the pharmacokinetics of these drugs in the vitreous are unknown, we hoped that these high concentrations would extend the duration of the drug effects as they were cleared from the vitreous. The amount injected ranged from 2.5 to $3.75 \mu \mathrm{l} ; 2.5 \mu \mathrm{l}$ was injected on P15 and the amount increased by $0.25 \mu \mathrm{l}$ for every other injected age (i.e., P17: $2.75 \mu \mathrm{l}$, P19: $3.0 \mu \mathrm{l}$, etc.) to compensate for the volume change of the growing eye. Injections were administered into the vitreous humor of each eye using a 32 gauge needle attached to a Hamilton microsyringe. Injections were made slowly over the course of several minutes to reduce sudden changes in intraocular pressure. Eyes were treated with antibiotic ophthalmic gel (Vetericyn; Innovacyn) after completion of the injection.

Extracellular recordings of visual responses in the dLGN. dLGN recordings were performed using protocols described previously (Chapman and Gödecke, 2000). Ferrets were anesthetized using a mixture of acepromazine $(0.4 \mathrm{mg} / \mathrm{kg})$ and ketamine $(40 \mathrm{mg} / \mathrm{kg}$, i.m. $)$, placed in a modified kitten stereotax, and paralyzed with vecuronium bromide $(0.2$ $\mathrm{mg} / \mathrm{kg} / \mathrm{h}$ ). Animals were intubated and anesthesia was maintained using $1-2 \%$ inhalant isoflurane in oxygen. End-tidal carbon dioxide, core body temperature, and electrocardiogram were monitored throughout the experiment. Atropine drops were administered to the eyes and the cornea was protected by a plano contact lens ( $4 \mathrm{~mm}$ diameter, base curvature 280; Futuristic Design Associates). We performed ophthalmic inspection of the cornea, lens, and retina to verify that there were no congenital defects or damage from the experimental procedures before recording from the ferrets. If such damage was found, then the animal was excluded from the experiment. A $4 \times 4 \mathrm{~mm}$ craniotomy was made over the dLGN and filled with agar. A tungsten electrode (1.5 $\mathrm{M} \Omega$; Micro Probe) was advanced through the agar into the dLGN using a microdriver and visual responses were measured to locate the dLGN. Once identified, single units were isolated with an audio monitor and oscilloscope and the receptive field size and location were mapped onto a tangent screen. Analyzed units had receptive field centers within $20^{\circ}$ of the area centralis as identified based on the reference location of the optic disk observed through an ophthalmoscope (Zahs and Stryker, 1985). Spike times and waveforms were recorded using a Spike 2 system (Cambridge Electronic Design). Spikes were sorted through waveform template matching in Spike 2. Single units were identified through PCA clustering of wave forms and the presence of a refractory period in the autocorrelogram.

In vivo visual stimulation. The functional properties of geniculate receptive fields were mapped through visual stimuli created using a VSG $2 / 5$ visual stimulator (Cambridge Research Systems) and displayed on a gamma-corrected Dell Trinitron CRT monitor with a mean luminance of $40-50 \mathrm{~cd} / \mathrm{m}^{2}$ and a refresh rate of $100 \mathrm{~Hz}$. The monitor was centered on the receptive field of the recorded unit at a distance of $57 \mathrm{~cm}$. Receptive field maps were calculated by reverse correlation from spike responses to a white noise stimulus (Reid et al., 1997). The white noise stimulus consisted of a $16 \times 16$ grid of squares that were white or black $50 \%$ of the time as determined by an m-sequence of length $2^{15}-1$. The size of the squares in the checkerboard and the rate at which the frames were presented varied, with sizes of squares ranging from 1.28 to $0.78 \mathrm{~cm}$ and the frequency of stimulus frames presented ranging from 100 to 25 $\mathrm{Hz}$. The parameters were chosen on a unit by unit basis based on the responses during hand mapping of the receptive field on the tangent screen to sufficiently sample the spatial and temporal dimensions of the receptive field.

Response latency and temporal integration were measured through 10 repetitions of an alternating full screen, maximum contrast, square-wave stimulus with a duration of $4 \mathrm{~s}$ per cycle. From this cells were characterized as On, Off, or mixed in their preferred response, the latency of their responses, and the strength of their transient onset, sustained, and offset 
responses. Finally, center surround structure was measured by the display of a drifting sine-wave stimulus of maximum contrast at a temporal frequency of $3-4 \mathrm{~Hz}$. The spatial frequencies presented ranged from low [minimum: 0.01 cycles per degree (CPD)] to high (maximum: $0.7 \mathrm{CPD}$ ) and a subset was selected based on the size of the receptive field to obtain spatial frequency-tuning curves.

Data analysis. Receptive fields were measured by calculating the spiketriggered average (STA) of the stimulus ensemble weighed by the number of spikes elicited by each frame of the m-sequence checkerboard stimulus as follows:

$$
S T A=\frac{1}{N} \sum_{n=1}^{N} S_{n}
$$

Where $N$ is the total number of spikes in the spike train and $S$ is the stimulus present for the $n$th spike of the spike train. The temporal response of the receptive field was measured by calculating the STA to each of 16 sequential stimulus frames preceding the $n$th spike. The peak frame of the temporal response was chosen and a bivariate Gaussian was fit to this frame as described by the following:

$$
\begin{aligned}
F(x, y)=A \exp \left(-0.5\left\{\left[\frac{(x-h) \cos \theta-(y-k) \sin \theta}{\sigma_{a}}\right]^{2}\right.\right. \\
\left.\left.+\left[\frac{(y-k) \cos \theta-(x-h) \sin \theta}{\sigma_{b}}\right]^{2}\right\}\right)
\end{aligned}
$$

Where $A$ is the amplitude of the Gaussian, $x$ and $y$ are independent spatial coordinates, $h$ and $k$ are the $x$ - and $y$-coordinate receptive field midpoints, respectively, $\sigma_{a}$ and $\sigma_{b}$ are the SDs of the major and minor axes respectively, and $\theta$ is the angle between the major axis of the Gaussian and the $x$-axes of the coordinate system (Cantrell et al., 2010).

Spatial frequency-tuning curves were normalized to the maximum amplitude of the frequency-modulated response and fit to the mean response amplitude at each spatial frequency using a difference of Gaussian function as follows:

$$
\mathrm{R}\left(\omega_{\mathrm{x}}\right)=k\left[\left(\exp -\left(\omega_{\mathrm{x}} / f_{c}\right)^{2}\right)-k_{s}\left(\exp -\left(\omega_{\mathrm{x}} / f_{s}\right)^{2}\right)\right],
$$

Where $\omega_{\mathrm{x}}$ is the spatial frequency, $k$ is a scaling constant, $f_{c}$ is the characteristic spatial frequency of the Gaussian representing the receptive field center, $k_{s}$ is the integrated weight of the surround relative to the center, and $f_{s}$ is the characteristic spatial frequency of the Gaussian representing the surround component of the receptive field. We take $f_{\mathcal{c}}$, a measure of the spatial resolution of the receptive field center, as the frequency at which the strength of the center mechanism has fallen to 1/e of its maximum. We take $1 / \pi f_{c}$ as the measure of receptive field center size (Enroth-Cugell and Robson, 1966; Movshon et al., 2005).

Multielectrode array recordings. Multielectrode array (MEA) recordings were performed as described previously (Warland et al., 2006; Sun et al., 2008; Davis et al., 2015). Ferrets were administered a lethal dose of pentobarbital $(0.1-0.2 \mathrm{ml})$ via intraperitoneal injection. For the retinal wave analysis, ferrets age P17-P18 and P25 were used; for the ganglion cell receptive field analysis, ferrets age P31 and P38 were used. The eyes were enucleated and the retinas were removed and stored in HEPESbuffered medium (Ames' medium; Sigma-Aldrich) and chilled on ice. A piece of retina was placed ganglion side down onto a 60-channel MEA (MultiChannel Systems) in a darkened recording chamber and held in place with a piece of dialysis membrane (Spectrapore 132130; Spectrum). The tissue was superfused with sodium bicarbonate buffered Ames' medium at $1-2 \mathrm{ml} / \mathrm{min}$ at $37^{\circ} \mathrm{C}$. The array electrodes were $30 \mu \mathrm{m}$ in diameter and arranged in an $8 \times 8$ rectilinear grid with an interelectrode spacing of $200 \mu \mathrm{m}$. At this distance, the signal for a given cell appeared on only one electrode, so each cell isolated was assigned the spatial coordinates of the electrode that recorded its signal. Analog data were acquired at $20 \mathrm{kHz}$ per channel simultaneously from each electrode. After the retina was set up on the MEA, the tissue was allowed to acclimate for 15-45 $\mathrm{min}$. On the emergence of retinal wave events, we observed the data for a period of minutes until the average frequency of waves and the average activity across the retina appeared stable. Recordings were then performed for 5-10 min. To verify the stability of our recordings, the first and last half of each recording was compared and no difference in spike, burst, or wave rate was found. The effects of CPPG and TGS on spontaneous retinal activity were measured by recording waves for $10 \mathrm{~min}$, adding either CPPG $(500 \mu \mathrm{M})$ or TPMPA $(50 \mu \mathrm{M})$, gabazine $(5 \mu \mathrm{M})$, and strychnine (500 nM) (TGS) to the perfusion system, and perfusing over the tissue for $5 \mathrm{~min}$. Waves were recorded for an additional $10 \mathrm{~min}$ at the normal perfusion rate after activity levels appeared stable.

Spike identification. Raw data were digitally filtered with a $125 \mathrm{~Hz}$ high-pass filter (four-pole Butterworth) sorting spike events. A threshold of 6 SDs was set for each channel and $1 \mathrm{~ms}$ of data before and $4 \mathrm{~ms}$ after a threshold-crossing event were stored for each negative-slope event. These candidate spike waveforms were then sorted with Offline Sorter (Plexon) using the first three principal components of the spike waveforms. Coincident events within $0.5 \mathrm{~ms}$ of each other that occurred on all electrodes were attributed to perfusion noise and removed. Clusters were first identified using an expectation-maximization (EM) cluster algorithm by Shoham et al. (2003) and then manually edited for clustering errors. Typically, the activity of one to three cells was recorded by each electrode.

Retinal wave analysis. The patterned activity of retinal waves and the consequences of activity perturbation via the introduction of pharmacological agents were examined in accordance with the analyses used by Stafford et al. (2009). A modified Poisson surprise algorithm was used to determine how frequently wave activity was present in the activity of MEA units. Using a $1 \mathrm{~s}$ bin, each single-unit spike train was converted to a time-varying spike count of $C$ spikes per bin and the mean spike rate $\lambda$ was calculated. Then, the probability, $P_{C i}$, that a Poisson spike train with a mean spike rate $\lambda$ would have $C$ spikes in time bin $i$ was calculated using the following equation:

$$
P_{C_{i}}=e^{-(\lambda)} \times \frac{(\lambda)^{C_{i}}}{C_{i} !}
$$

We then identified bins where the probability that a Poisson spike train would generate that spike count was $<10^{-5}$ and classified the unit to be bursting at that time. We identified the retina to be undergoing a retinal wave when $>10 \%$ of all of the units in the recording were bursting in the same time bin. Wave activity had ended when $<5 \%$ of the neurons were still bursting. The result is a count of time bins for which some wave activity was present in the recording. This algorithm was well suited for identifying the presence of waves in both normal and drug-excited levels of activity compared with estimates made by human observers.

Burst analysis. The bursting activity of MEA units was calculated independently of the wave analysis using a modified version of the Poisson surprise algorithm detailed above (Stafford et al., 2009). Here, bursts were classified for groups of three or more spikes that occurred within a time window smaller than expected based on the average spike timing of the unit. Instead of bins, the mean interspike interval (ISI), $\partial$, was calculated for each unit. When at least three sequential spikes were found such that their mean ISI $<\partial / 2$, the algorithm calculated the probability, $P_{C_{i}}$, that a unit with a mean spike rate, $\lambda$, would have $C_{\mathrm{i}}$ spikes in the time bin $T$ as determined by the duration between the first and last spike using the following equation:

$$
P_{C_{i}}=e^{-(\lambda \times T)} \times \frac{(\lambda \times T)^{C_{i}}}{C_{i} !}
$$

The spikes were classified as part of a burst if $P_{C_{i}}$ was $<10^{-4}$. Once a burst was identified, spikes were added to $C_{i}$ and the calculation repeated until either $P_{C_{i}}$ exceeded the initial value or the ISI of the next spike was greater than $\partial$ after the preceding spike.

Correlation analysis. The degree of correlated firing between pairs of recorded cells were calculated with cross-correlation functions and assigned a correlation index (CI). The CI measures the likelihood relative to chance that a pair of cells fired together within a given time window. The CI was computed as described by Wong et al. (1993) using the following equation: 
a White Noise Stimulus
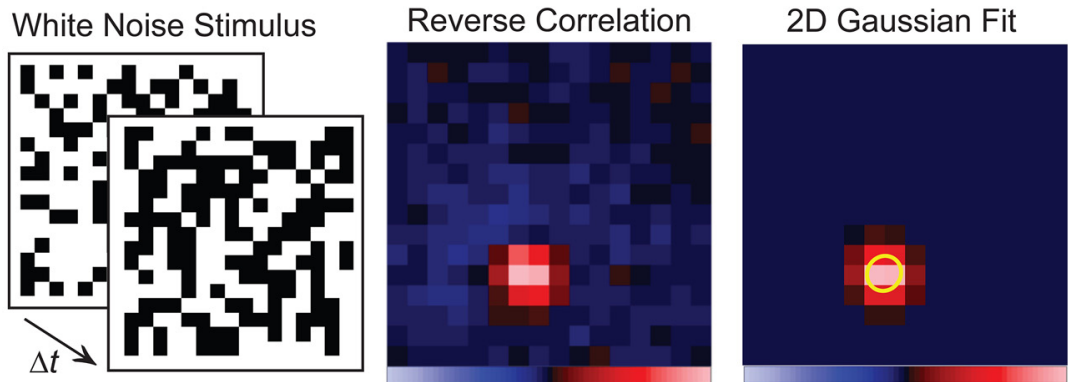

Excited by dark Excited by light Excited by dark Excited by light
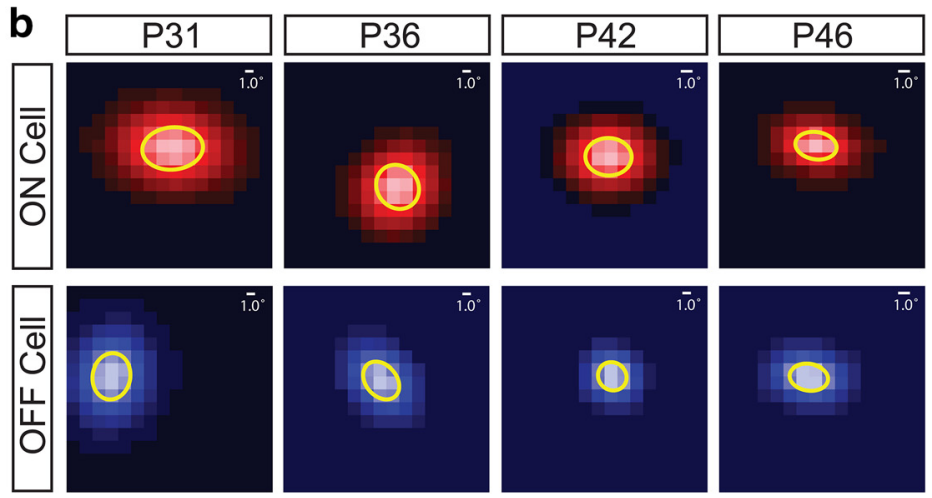

C

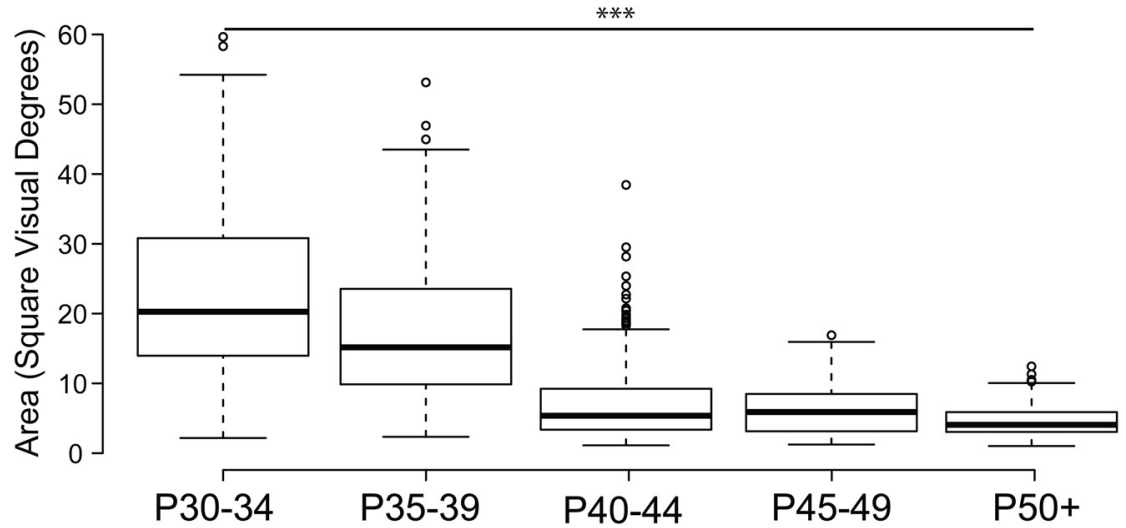

Figure 1. Ferret geniculate receptive fields are refined over the first postnatal month after eye opening. $\boldsymbol{a}$, Receptive fields of geniculate neurons in ferrets during the second postnatal month were measured by calculating the STA from the reverse correlation of a white noise stimulus. Red squares represent stimulus locations where the unit was excited by increasing luminance and the blue squares represent stimulus locations excited by decreasing luminance. The peak frame of the response was selected and a 2D Gaussian was fit to the receptive field center. The SD of the major and minor axis of the Gaussian was used to define the spatial area of the receptive field. $\boldsymbol{b}$, Representative Gaussian fits of $0 \mathrm{n}$ and 0 ff selective single unit receptive fields from control ferrets treated with intraocular saline injections from P15-P25. The 1SD ellipse is highlighted in yellow. Scale bars indicate the size of 1 visual degree relative to the size of each receptive field. $\boldsymbol{c}$, Receptive fields become significantly smaller in the weeks after eye opening. Box-and-whisker plots are of all units recorded across all saline-treated ferrets grouped in $5 \mathrm{~d}$ windows after P30. Center lines show the medians; box limits indicate the 25 th and 75 th percentiles; whiskers extend 1.5 times the interquartile range from the 25 th and 75 th percentiles; and outliers are represented by dots $\left({ }^{* * *} p<0.0001\right)$.

$$
C I=\frac{\mathrm{N}_{\mathrm{ab}(-\mathrm{w},+\mathrm{w})} \times \mathrm{T}}{\left[\mathrm{N}_{\mathrm{a}(0, \mathrm{~T})} \times \mathrm{N}_{\mathrm{b}(0, \mathrm{~T})} \times(2 \times \mathrm{w})\right]}
$$

Where $\mathrm{N}_{\mathrm{ab}(-\mathrm{w},+\mathrm{w})}$ is the number of spike pairs from cells $a$ and $b$ for which cell $b$ fires within $w$ seconds of cell $a, T$ is the duration of the recording in seconds, $\mathrm{N}_{\mathrm{a}(0, \mathrm{~T})}$ and $\mathrm{N}_{\mathrm{b}(0, \mathrm{~T})}$ are the total number of spikes from cell $a$ and $b$ during the recording, and $2 \times w$ is the width of the correlation window. $\mathrm{N}_{\mathrm{ab}}$ was computed using $w=0.1 \mathrm{~s}$ and the cross-correlation function was binned at $0.05 \mathrm{~s}$.

We also calculated the spike time tiling coefficient (STTC) because it has been shown that $\mathrm{CI}$ is influenced by the firing rates of the neurons in
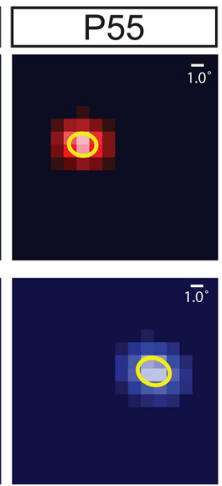

the recording and our experimental manipulations differentially influence firing rates, which could confound our CI analysis (Cutts and Eglen, 2014). We used the STTC equation as follows:

$$
S T T C=\frac{1}{2}\left(\frac{P_{A}-T_{B}}{1-P_{A} T_{B}}+\frac{P_{B}-T_{A}}{1-P_{B} T_{A}}\right)
$$

Where $P_{A}$ is the proportion of spikes in spike train $A$ that lie within $\pm \Delta t$ of any spike from $B$ and $T_{A}$ is the proportion of total recording time that lies within $\pm \Delta t$ of any spike from $A$. $P_{B}$ and $T_{B}$ are calculated similarly.

Retinal visual stimulation. Ganglion cell receptive fields were mapped through the reverse correlation of the same $m$-sequence presentation of a $16 \times 16$ grid of black and white squares used in the in vivo recordings. The visual stimuli were projected by a Dell projector (M109) through a dissection lens mounted over the projector into a microscope housing the recording chamber and reflected to project up through the bottom of the array onto the retina. The projector had a refresh rate of $60 \mathrm{~Hz}$ and the image was passed through a neutral density filter to arrive with a mean luminance of $10 \mathrm{~cd} / \mathrm{m}^{2}$. The image was focused by adjusting the distance of the dissection lens and by adjusting the optics of the microscope to bring the image into focus on the plane of the MEA. The images of the $m$-sequence were presented at a rate of 30 or $60 \mathrm{~Hz}$ and the size of each box in the $16 \times 16$ grid was varied between 80 and $110 \mu \mathrm{m}$ (or $\sim 0.75-1^{\circ}$ visual angle) across the MEA, depending on the age of the retina, to allow for the entire grid to sample both within the receptive fields diameters of individual ganglion cells $(\sim 200-300 \mu \mathrm{m})$ and across the entire area of recorded tissue $(\sim 1600 \mu \mathrm{m})$. MEA recordings were performed as described previously and all receptive field analyses were performed using the same methods as the in vivo recordings.

\section{Results}

Ferret eye opening occurs after the fourth postnatal week, at which time the receptive fields of geniculate neurons are large and immature (Tavazoie and Reid, 2000). The majority of refinement occurs during the second postnatal month, with mature adult receptive fields present by the third postnatal month (Tavazoie and Reid, 2000). Control ferrets were intraocularly injected with sterile saline daily from P15P25 during a period of glutamatergic retinal waves. The receptive field properties of geniculate neurons were characterized by reverse correlation of an $m$-sequence checkerboard stimulus (see Materials and Methods) at ages between eye opening around P30 and the end of the second postnatal month. We quantified the size of the receptive field defined as the SD of the 2D Gaussian fit to the peak of the spatiotemporal receptive field (Fig. 1a). All receptive fields were recorded from within $20^{\circ}$ of the center of visual space from the contralateral A lamina of the dLGN (typically $0-10^{\circ}$ from center) and were made from X-like cells in which classification was possible based on the 
response dynamics to a full field-flashing stimulus. All ferrets recorded had similar distributions of receptive field eccentricity. There was no difference in the development of On or Off cells, so both were grouped together for this analysis.

Similar to previous studies on the development of ferret geniculate receptive fields (Tavazoie and Reid, 2000), we found that the majority of refinement and maturation occurs in the month after eye opening (Fig. 1b,c). Around eye opening at P30, salinetreated ferret geniculate neurons responded weakly and were difficult to isolate and classify. Of those that we could characterize, the receptive fields had a mean area of $25 \pm 3$ (SEM) square visual degrees (293 units from 7 ferrets age P30-P34). By the end of the fifth postnatal week, the mean receptive field area had decreased by $32 \%$ to $17 \pm 2$ (SEM) square visual degrees (163 units from 3 ferrets age P35-P39). At the end of the sixth postnatal week, receptive fields had further decreased by $56 \%$ with a mean area of $7.5 \pm 1.6(\mathrm{SEM})$ square visual degrees (281 units from 6 ferrets age $\mathrm{P} 40-\mathrm{P} 44$ ). The mean receptive field size stayed stable through the end of the seventh postnatal week $[7.9 \pm 1.3$ (SEM) square visual degrees (531 units from 4 ferrets age P45-P49)] until reaching more adult-like sizes around the end of the second postnatal month $[4.6 \pm 0.5$ (SEM) square visual degrees (260 units from 3 ferrets age P50+)]. Overall, there was a significant decrease in receptive field size during the postnatal month after eye opening (one-way ANOVA, $F_{(4,17)}=14.83 ; p<0.0001$ ). Our results nicely replicate previous experiments characterizing the development of ferret geniculate receptive fields after eye opening (Tavazoie and Reid, 2000).

Response latency was also measured from the peristimulustime histograms of visual responses to a full field stimulus presented as a square wave with a frequency of $0.25 \mathrm{~Hz}$. The histogram was binned at $5 \mathrm{~ms}$ and the response latency was taken as the first time bin to contain more spikes than expected $95 \%$ of the time given the mean spontaneous firing rate as calculated from a prestimulus baseline. At eye opening, the mean latency of geniculate neurons was $72 \pm 7$ (SEM) ms. After a week of normal visual experience, the latency decreased by $36 \%$ to $46 \pm 1$ (SEM) ms and gradually declined until the end of the second postnatal month to a mean latency of $38 \pm 1(\mathrm{SEM}) \mathrm{ms}$. There was a significant reduction in geniculate response latency between eye opening around P30 and the end of the second postnatal month $(p=0.009)$.

\section{Increased spontaneous activity decreases receptive field size at eye opening}

Normal visual experience drives the refinement of geniculate receptive fields. We wished to investigate whether increasing the spontaneous activity of the retina before visual experience could also drive the refinement of geniculate receptive fields. We administered daily injections of either CPPG, an mGluR6 antagonist that excites On bipolar cells and increases spontaneous activity (Awatramani and Slaughter, 2000), or a combination of the inhibitory antagonists TPMPA, gabazine, and strychnine (TGS), which results in increases in spontaneous activity and has been used previously to influence retinal activity (Kerschensteiner and Wong, 2008; Firl et al., 2013). The drugs were injected from P15-P25, ages corresponding to a period of late glutamatergic retinal waves before eye opening in the ferret. We then waited until $\mathrm{P} 30$, the age of natural eye opening in the ferret, and evaluated the size of geniculate receptive fields of CPPG- and TGS-treated ferrets in the same manner as saline controls (Fig. 2a).

To compare the consequence of drug treatment on geniculate receptive field size at different ages of development (Fig. $2 b, c$ ), we performed a mixed-model ANOVA of unit by ferret by condition with Tukey's (HSD) multiple-comparison tests for post hoc interactions of experimental condition and ferret age. At eye opening, the receptive fields of TGS- and CPPG-treated ferrets were not significantly different from each other and both treatments resulted in receptive fields that were significantly smaller than saline-treated controls $(p<0.0001$ and $p<0.0001$, respectively; Fig. $2 d$ ). The mean receptive field size in TGS-treated ferrets was $40 \%$ smaller than controls at $15 \pm 1$ (SEM) square visual degrees (285 units from 5 ferrets age P30-P34) and was similarly smaller for CPPG-treated ferrets [14 \pm 2 (SEM) square visual degrees; 320 units from 5 ferrets age P30-P34]. However, despite this difference in receptive field size, there was no difference in the mean latency of visual responses between either TGS treatment $(p=0.54)$ or CPPG treatment $(p=0.87)$ and saline treatment.

There was no difference in the geniculate receptive field size of ferrets in the fifth postnatal week or later between saline and TGS-treated $(p=0.27)$ or CPPG-treated $(p=0.11)$ ferrets. Although receptive fields for the drug-treated groups were unchanged between eye opening and the end of the fifth postnatal week [16 \pm 4 (SEM); 234 units from 4 TGS-treated ferrets age P35-P39; $13 \pm 1$ (SEM) square visual degrees; 222 units from 3 CPPG-treated ferrets age P35-P39], there was the same progression in the reduction in geniculate receptive field sizes with visual experience observed in saline controls.

\section{Mechanism of receptive field refinement}

Experimentally increasing spontaneous activity during glutamatergic retinal waves reduced the size of geniculate receptive fields at eye opening. There are at least two ways in which increased activity can produce this effect. First, the increase in activity could drive Hebbian mechanisms of plasticity and cause refinement of convergent inputs from numerous RGCs spanning a larger retinotopic area while strengthening the inputs driving the center of the receptive field. The increase in activity could also accelerate the development and maturation of surround mechanisms that could serve to suppress the flanks of the receptive field center.

To compare the relative contribution of center and surround mechanisms to the receptive field of geniculate neurons after eye opening, we measured the spatial frequency tuning of a subset of recorded neurons to increasing spatial frequencies of a drifting sine-wave grating and fit the amplitude modulated responses to a difference of Gaussian function (Enroth-Cugell and Robson, 1966; Fig. 3a). TGS- and CPPG-treated ferrets were combined because there was no observed difference in their receptive field properties. At P30, saline-treated ferrets had a mean characteristic spatial frequency of $0.07 \pm 0.01$ (SEM) CPD (158 units from 6 ferrets age P30-P34). This was significantly smaller than animals undergoing drug treatment during glutamatergic retinal waves $[0.1 \pm 0.01$ (SEM) CPD; 326 units from 7 ferrets age P30-P34 $p<0.001$; Fig. $3 b]$.

From the spatial frequency tuning of our geniculate neurons, we estimated the receptive field size (Movshon et al., 2005; Fig. $3 c$ ) and compared the results with our estimates from the reverse correlation of the white noise stimulus. Saline-treated ferrets had an estimated receptive field of $20 \pm 2$ (SEM) square visual degrees and drug-treated ferrets had a significantly smaller estimated receptive field size of $11 \pm 1$ (SEM) square visual degrees after eye opening $(p<0.001)$. Importantly, these values corroborate our estimates of receptive field size from the reverse correlation of our white noise stimulus, which gave mean estimates of 25 and 14 square visual degrees, respectively.

We then investigated whether surround suppression differs between saline- and drug-treated ferrets by examining the presence 

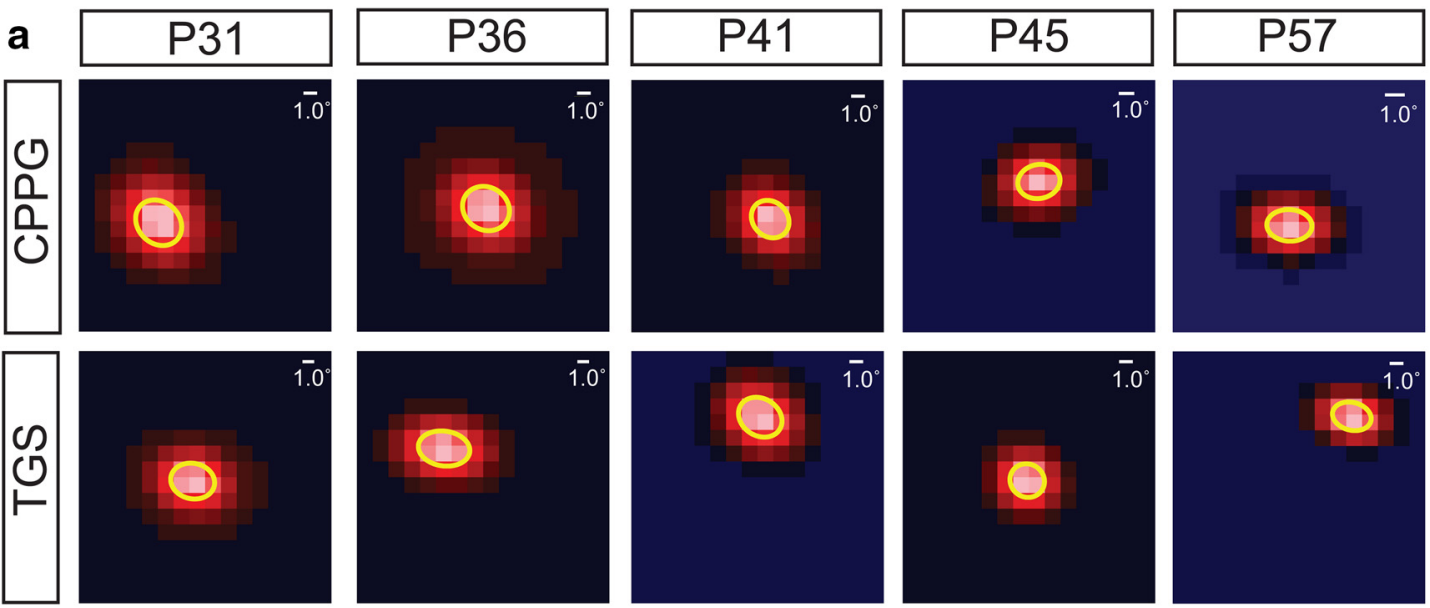

b CPPG Treated Receptive Field Development d
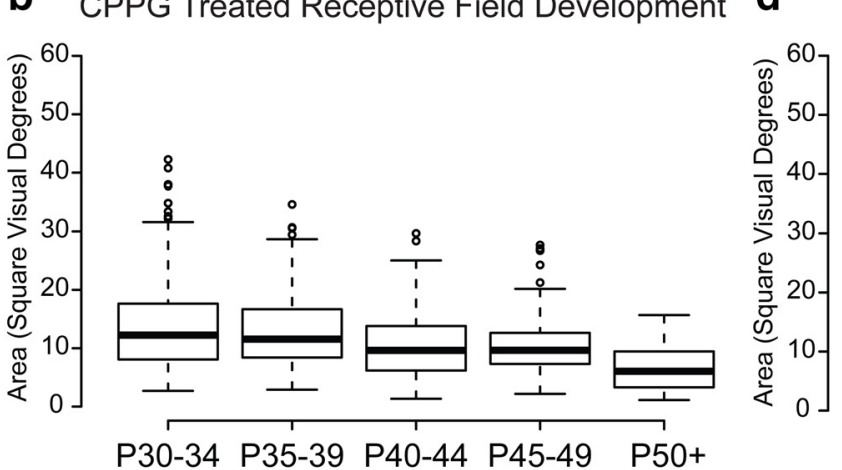

P30-34 Receptive Field Area

C TGS Treated Receptive Field Development

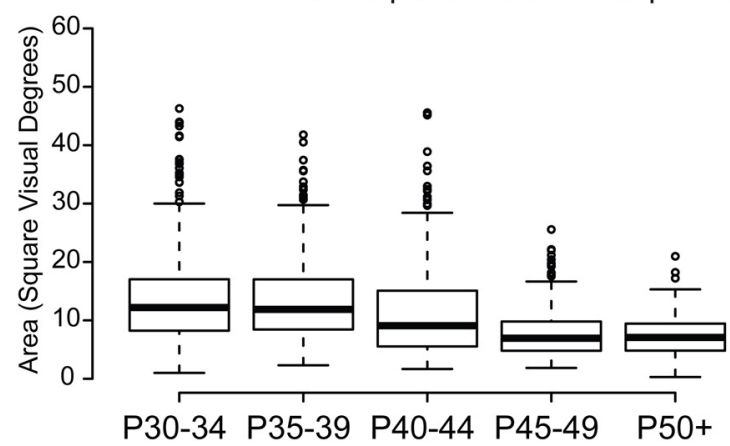

Figure 2. Experimentally increased spontaneous retinal activity before eye opening drives a reduction in geniculate receptive field size before visual experience. $\boldsymbol{a}, 2 \mathrm{D}$ Gaussian fits from representative receptive fields of ferrets treated with intraocular injections of the mGluR6 antagonist CPPG (2 mM) (top row) or a combination of TPMPA ( $2 \mathrm{mM})$, gabazine (200 $\mu \mathrm{M})$, and strychnine $(20 \mu \mathrm{M})$ [TGS] (bottom row) from P15-P25 are shown from the second postnatal month of development. Scale bars indicate the width of 1 visual degree relative to the size of the receptive fields. $\boldsymbol{b}, \boldsymbol{c}$, Developmental refinement of geniculate receptive field sizes in CPPG- and TGS-treated ferrets, respectively. Box-and-whisker plots are formatted the same as in Figure 1. There is no difference between the two treatment groups. $\boldsymbol{d}$, Box-and-whisker plot illustrating the significant difference between receptive field sizes of saline-treated ferrets and both CPPG- and TGS-treated ferrets at eye opening $\left({ }^{* * *} p<0.001\right)$.

and strength of surround inhibition in the spatial frequency-tuning curves. Units with no surround inhibition have low-pass spatial frequency-tuning curves, whereas cells with surround inhibition have band-pass spatial frequency-tuning curves. Surround strength was calculated as the maximum suppression in the response amplitude for spatial frequencies lower than the peak spatial frequency for units that showed some degree of surround suppression.

At eye opening, ferret geniculate surround mechanisms were largely absent from the receptive fields and very weak when detected (Fig. $3 d$ ). There was no difference in the percentage of cells that displayed surround suppression at low spatial frequencies $[21 \pm 5(\mathrm{SEM})$ percent in saline-treated vs $23 \pm 5$ (SEM) percent in drug-treated, $(p=0.98)]$, nor was there a difference in the strength of surround suppression observed in the units that did have it $[20 \pm 2($ SEM) percent in saline-treated vs $21 \pm 5$ (SEM) percent in drug-treated $(p=0.42)]$. Over the course of visual development, surround mechanisms became stronger. There was a significant increase in the strength of surround suppression with age for both saline-treated ferrets (86\% increase; $p<$ $0.0001)$ and drug-treated ferrets (105\% increase; $p<0.0001)$. However, there was no difference in the rate of development of either the frequency of cells with surrounds or the strength of surrounds between the two drug treatment groups $(p=0.91)$. From this, we conclude that the consequence of increased spon- 

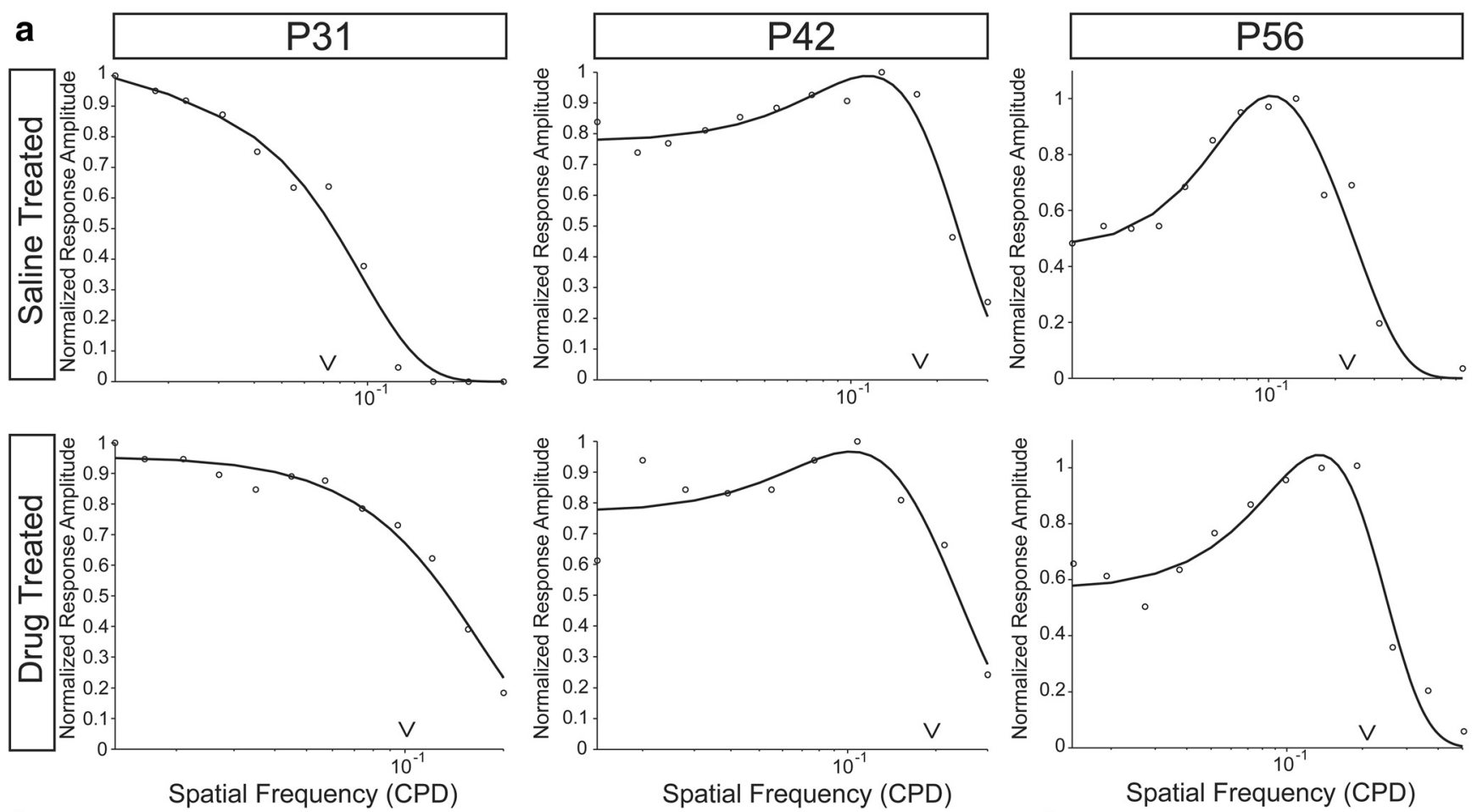

b

\section{C}
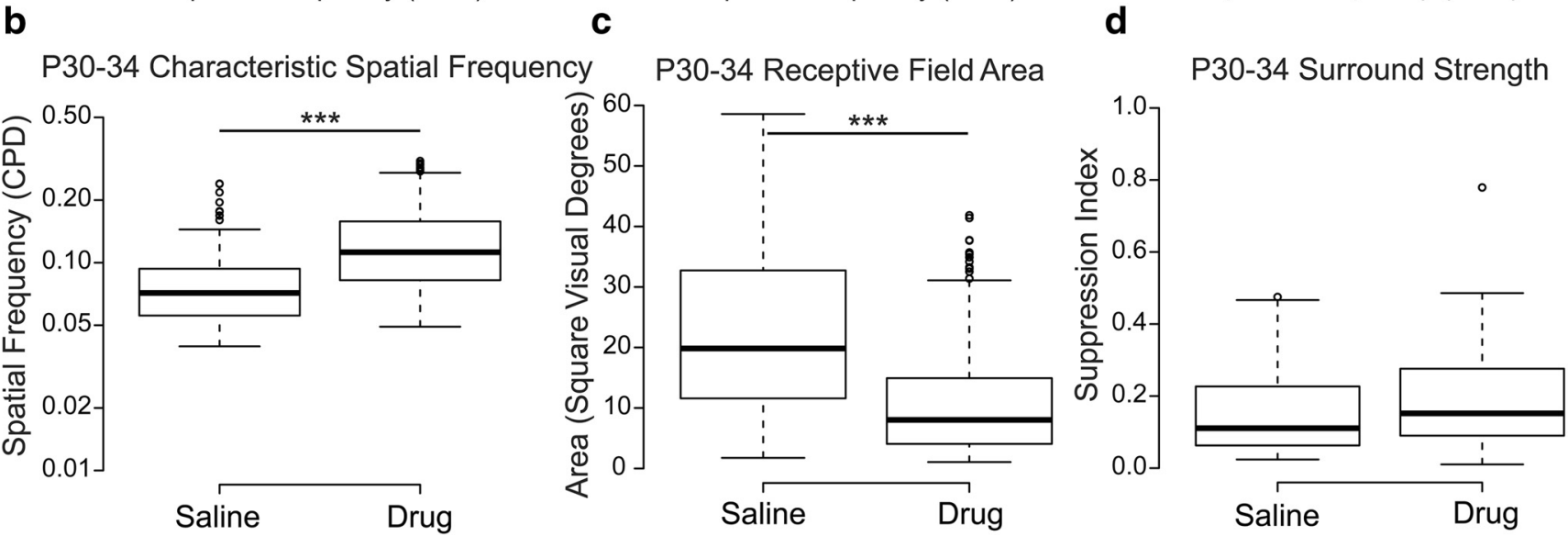

P30-34 Surround Strength

Figure 3. Characteristic spatial frequency, but not surround suppression, differs between saline-treated and drug-treated ferrets after eye opening. $\boldsymbol{a}$, Representative spatial frequency-tuning curves from saline-treated (top) and either TGS- or CPPG-treated ferrets (drug-treated, bottom). Carets indicate the characteristic spatial frequency of the tuning curve as defined by the value where the response amplitude has fallen off to $1 /$ from the peak response. Tuning curves are more low-pass after eye opening, suggesting little surround suppression of responses to low spatial frequency stimuli. At older ages, tuning curves are more band-pass, indicating surround suppression of stimuli larger than the width of the receptive field center. $\boldsymbol{b}$, Box-and-whisker plots showing a significant difference in the characteristic spatial frequency between saline-treated and drug-treated ferret receptive fields after eye opening. $\boldsymbol{c}$, Receptive field areas calculated from the characteristic spatial frequency show a significant difference between saline- and drug-treated ferrets and closely resemble the estimates obtained from reverse correlations of the white noise stimulus. $\boldsymbol{d}$, Few units show suppressive surrounds in either saline- or drug-treated ferrets after eye opening and the suppression that we observe is comparatively weak in both conditions, suggesting that receptive field differences are not due to differences in surround inhibition $\left({ }^{* * *} p<0.001\right)$.

taneous activity during glutamatergic retinal waves is a reduction in the convergent input to the center of the geniculate receptive field and not a change in the strength of surround inhibition.

\section{Drug application increases patterned spontaneous retinal activity}

To approximate the consequence of our drug treatment on the properties of spontaneous retinal waves in vivo, we acutely recorded from retinal tissue using a MEA before and after the introduction of either CPPG or TGS. We could not measure the in vivo injection's effect on activity because the drugs used quickly washed out of the retina after extraction and perfusion on the MEA and no evidence of altered spontaneous activity was observed in retinas extracted $24 \mathrm{~h}$ after injection. Therefore, we approximated the intraocular concentration from the volume of the ferret eye and recorded at this diluted concentration (500 $\mu \mathrm{M}$ CPPG or $50 \mu \mathrm{M}$ TPMPA, $5 \mu \mathrm{M}$ gabazine, and $500 \mathrm{nM}$ strychnine). Spontaneous retinal activity was greatly increased in the presence of the treatment drugs (Fig. 4a). Units from each retina were averaged together and statistical significance was measured across retinas recorded with an ANOVA and pairwise analyses were made with a post hoc Tukey's (HSD) test for multiple comparisons.

CPPG and TGS application significantly increased the spontaneous spike and burst rates from $2.1 \pm 0.2$ (SEM) spikes/s (Fig. 4b) and $2.3 \pm 0.3$ (SEM) bursts per minute (744 units from 10 retinas P17P18) (Fig. 4c) to $8.2 \pm 0.6$ (SEM) (284\% increase) spikes/s and $5.1 \pm$ 


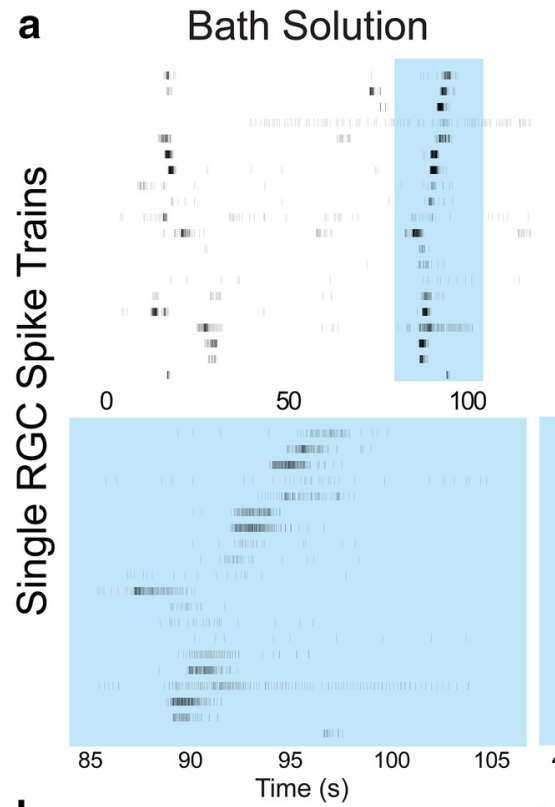

b

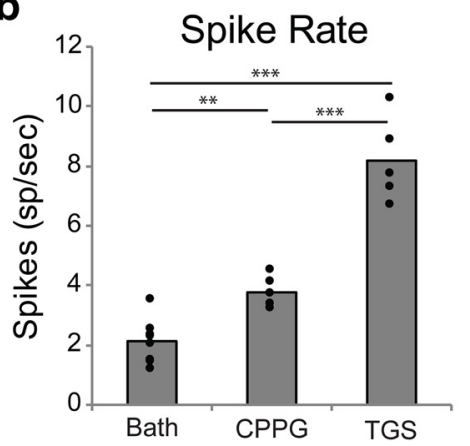

CPPG
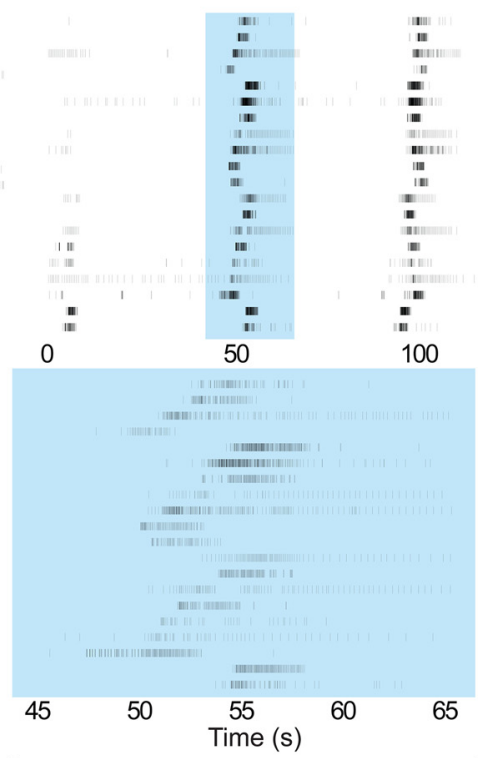

c

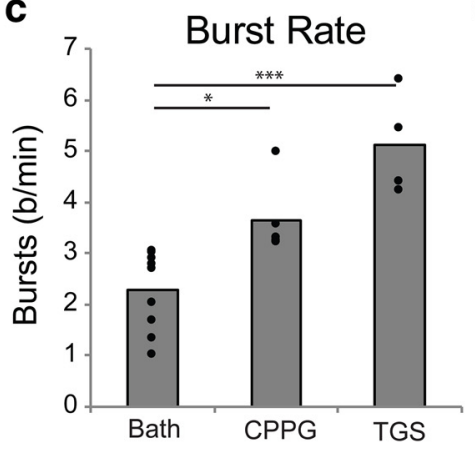

TGS

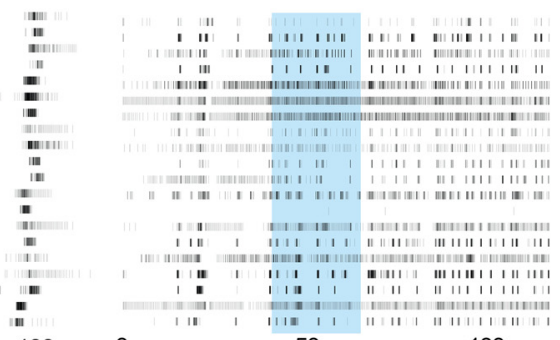

50

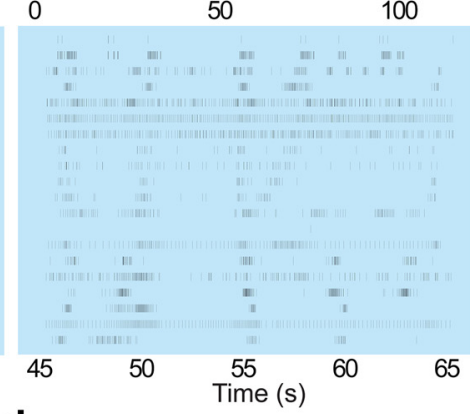

d

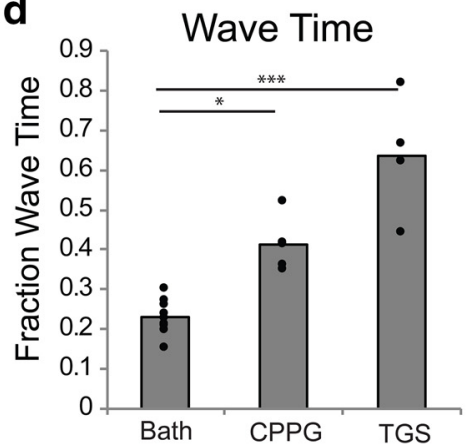

Figure 4. Drug application increases the spontaneous activity of the retina. $\boldsymbol{a}$, MEA recordings of P17-P18 RGCs in bath solution, CPPG (500 $\mu \mathrm{M})$, or TGS [TPMPA (500 $\mu \mathrm{M})$, gabazine (50 $\mu \mathrm{M})$, and strychnine $(5 \mu \mathrm{M})]$. Each row in the raster plot is a spike train from a single unit arranged vertically based on the location of its recording electrode on the MEA relative to the other units. Units that are adjacent to each other were recorded from the same or adjacent electrodes. Recording times highlighted in blue in the top raster plot are shown on a shorter time line on the bottom raster plot. Both CPPG and TGS application increased the firing rates $(\boldsymbol{b})$, bursting rates $(\boldsymbol{c})$, and percentage of time that wave activity was present $(\boldsymbol{d})$ compared with baseline rates. Bar graphs show the mean values for each condition and individual retinal recordings are plotted as solid circles on the bar graph $\left({ }^{*} p<0.05\right.$; $\left.{ }^{* *} p<0.01,{ }^{* * *} p<0.001\right)$.

0.5 (SEM) (125\% increase) bursts per minute with TGS $(p<0.001$ and $p<0.001$, respectively; 245 units from 5 retinas P17-P18) to $3.8 \pm 0.2(\mathrm{SEM})(77 \%$ increase) spikes/s and $3.7 \pm 0.3(\mathrm{SEM})(61 \%$ increase) bursts per minute with CPPG $(p=0.013$ and $p=0.047$, respectively; 385 units from 5 retinas at $\mathrm{P} 17-\mathrm{P} 18)$.

Retinal wave activity was detected using the methods outlined in Stafford et al. (2009). The amount of wave activity present in the recordings significantly increased from $23 \pm 1$ (SEM) percent of recording time (Fig. $4 d$ ) to $64 \pm 7$ (SEM) percent of recording time with TGS $(p<0.0001)$ and $41 \pm 3(\mathrm{SEM})$ percent of recording time with CPPG $(p<0.03)$. There was no difference between CPPG and TGS on the magnitude of wave time increase $(p=0.112)$.

To determine whether the increase in spiking activity caused by the drug application could alone account for our increased measurement of wave activity, Poisson-distributed spike trains were added to baseline spontaneous recordings to increase the mean spike rate to levels observed after TGS application. Mean firing rates were increased from $2.1 \pm 0.2$ (SEM) spikes/s at baseline to $8.2 \pm 0.3$ (SEM) spikes/s. Increasing the spike rates decreased the amount of time that waves were observed by nearly $25 \%$, from $23 \pm$ $1 \%$ (SEM) of the time to $17 \pm 2 \%$ (SEM) $(p<0.03)$. This is likely due to a failure to classify cells as bursting or waving when the background firing rate becomes more similar to rates during wave activ- ity. Therefore, the observation of increased wave activity after drug treatment is most likely due to a real increase in wave activity and not a consequence of increased spike rates.

To investigate the consequence of chronic exposure of CPPG and TGS on the spontaneous activity of the retina, we extracted retinas at P25 after $10 \mathrm{~d}$ of saline, CPPG, or TGS injection (and $24 \mathrm{~h}$ after the final injection) and recorded in normal bath solution. There was no difference in the spike rate (CPPG: $p=0.62$; TGS: $p=0.34$ ) or burst rate (CPPG: $p=0.99$; TGS: $p=0.46$ ) between retinas extracted from saline-treated or drug-treated conditions, suggesting that the effects of these drugs were transient and did not result in permanent changes to the spontaneous activity of the retina. Either CPPG or TGS application to the bath at P25 still produced increases in spike rate and burst rate similar to earlier time points.

Correlations in the spatiotemporal structure of spontaneous retinal waves have been implicated as the instructive signal driving the development of the retinogeniculate circuit (Butts and Rokhsar, 2001; Stafford et al., 2009). To investigate whether our activity increase disrupted correlated activity, we calculated the correlation index (CI) (Wong et al., 1993) as a function of retinal distance on the array (Fig. $5 a$ ). Units were highly correlated at distances up to $400 \mu \mathrm{m}$ in the normal spontaneous activity of our MEA recordings, with a maximum CI of $19.6 \pm 2.6$ (SEM). Al- 
a

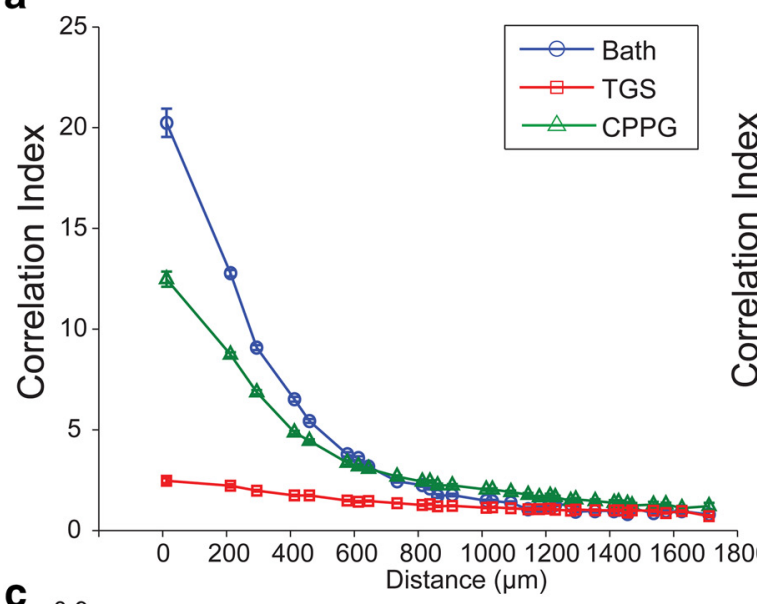

C

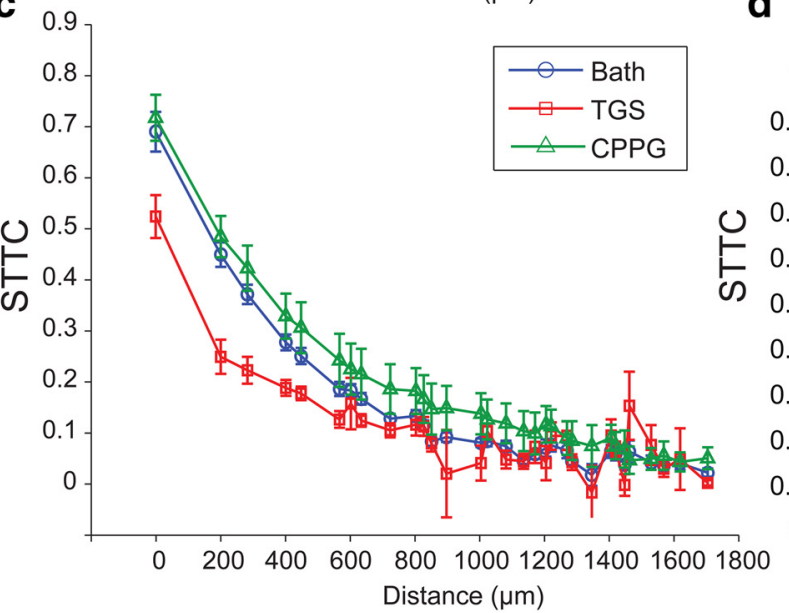

b

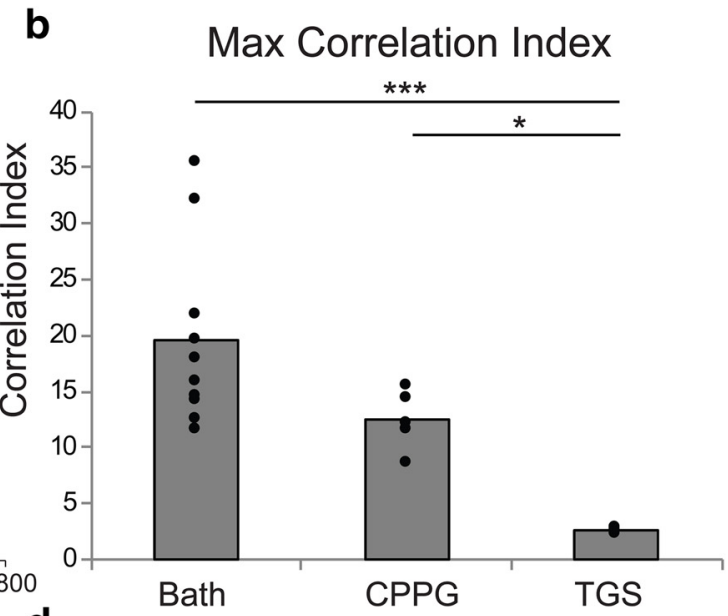

d

Figure 5. Correlations in the spontaneous activity of the retina persist after drug application. $\boldsymbol{a}, \mathrm{Cl}$ plotted as a function of interunit distance on the MEA for retinas recorded in bath solution (blue circles), TGS (red squares), or CPPG (green triangles). Data points are mean values for all units recorded from all retinas; error bars indicate SEM. CPPG application modestly reduces the CI and TGS application almost entirely abolishes the relationship between $\mathrm{Cl}$ and distance on the MEA. $\boldsymbol{b}$, Maximum CI was significantly reduced with both CPPG and TGS. Bars are the mean value of the maximum Cl from across retinas; the filled circles are the maximum Cl of each retina recorded. TGS application significantly reduces the max CI compared with both bath and CPPG application. $C$, STTCS are plotted the same as for the Cl in $\boldsymbol{a}$. STTC removes bias in estimating correlated activity during retinal waves due to differences in firing rate. CPPG does not reduce STTC from bath solution and TGS only modestly reduces STTC. $\boldsymbol{d}$, There is no significant difference in the maximum STTC among bath, CPPG, and TGS across retinas, suggesting that the loss of correlation observed in the Cl analysis is due to the higher spike rates $\left({ }^{*} p<0.05,{ }^{* * *} p<0.001\right)$. For reference, $200 \mu \mathrm{m}$ subtends $\sim 2.3^{\circ}$ visual angle at the ages recorded.

though there was a significant reduction in maximum CI with CPPG application [12.4 $\pm 1.2(\mathrm{SEM}), p<0.003$ ], the spatiotemporal pattern of correlations was still present in the spiking activity. In contrast, the large increase in spiking activity as a consequence of TGS treatment severely decorrelated the pattern of activity, with a maximum CI of only $2.4 \pm 0.1$ (SEM), which was significantly less than both bath $(p<0.0001)$ and CPPG $(p=0.018)$ and resulted in no relationship between CI and retinal distance (Fig. $5 b$ ).

Recently, it has been demonstrated that the CI measure is confounded by the relative firing rates of the units used in the analysis (Cutts and Eglen, 2014). Because our experimental manipulation increases firing rates, we wished to dissociate changes in CI from changes in firing rate by analyzing the correlations in waves using the spike time tiling coefficient (STTC) (Cutts and Eglen, 2014). This measure provides a firing-rate-independent estimate of the correlated firing between pairs of units and is bounded by 1 for perfectly correlated, -1 for perfectly anticorrelated, and 0 for uncorrelated. The results of our STTC analysis mirrored the local correlations observed from the CI analysis for bath and CPPG conditions (Fig. $5 c$ ). Spontaneous retinal waves were highly correlated in their temporal firing over small distances $[\mathrm{STTC}=0.70 \pm 0.04(\mathrm{SEM})]$. CPPG did not produce a decrease in correlations compared with baseline, suggesting that the reduction of correlation observed with the CI analysis was due to the increased firing rate $[\mathrm{STTC}=0.73 \pm 0.05(\mathrm{SEM}) ; p=$ $0.91]$. TGS treatment also did not yield a significant decrease in local correlations, but there was a trend toward less correlation compared with baseline [STTC $=0.53 \pm 0.05(\mathrm{SEM}) ; p=0.054]$ (Fig. $5 d$ ). Regardless, the STTC analysis suggests that there were persistent local correlations in the structure of the activity of TGS-exposed retinas that could be sufficient to drive refinement.

We conclude that CPPG and TGS increase the spiking and bursting activity of the retina, as well as the amount of time retinal waves propagate through the retina, and these activities retain the correlated information believed to be important for development in the visual system.

\section{Increased retinal activity does not decrease $\mathrm{RGC}$ receptive field size}

One possible explanation for the decrease in geniculate receptive field size is that the increase in spontaneous retinal activity instead reduces the size of RGC receptive fields and the dLGN inherits smaller receptive fields from the retina. To test this hypothesis, we measured the receptive field sizes of RGCs on an 

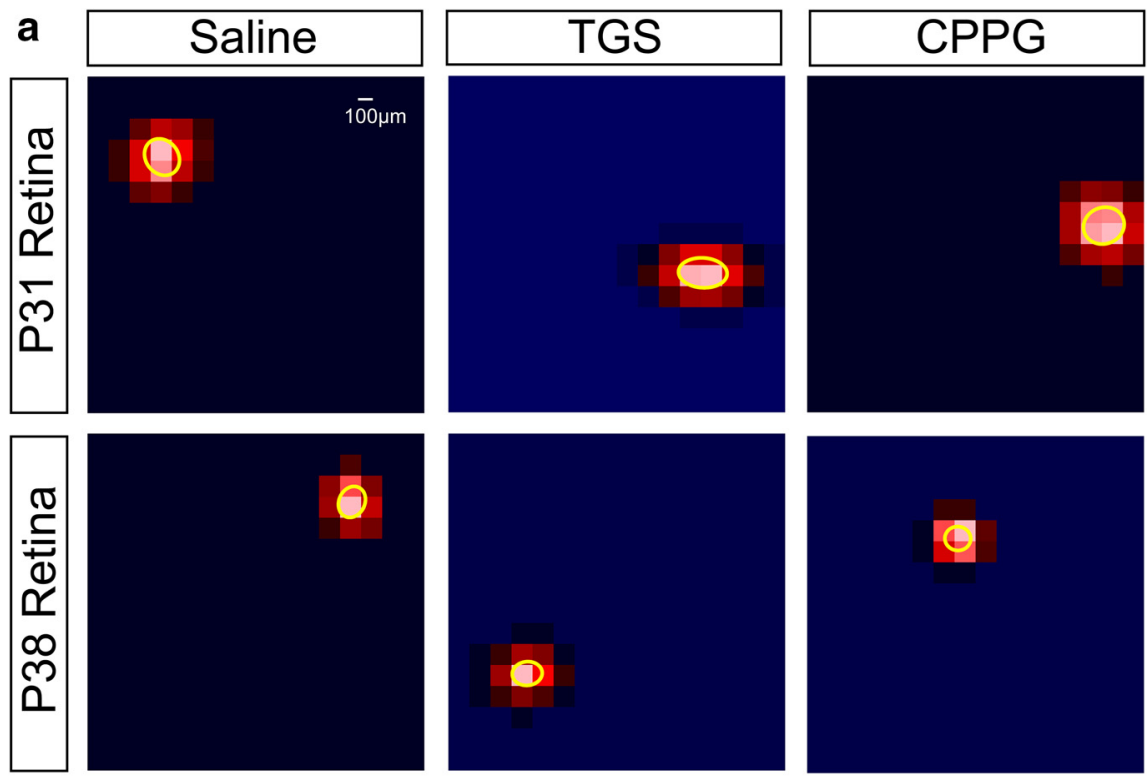

b

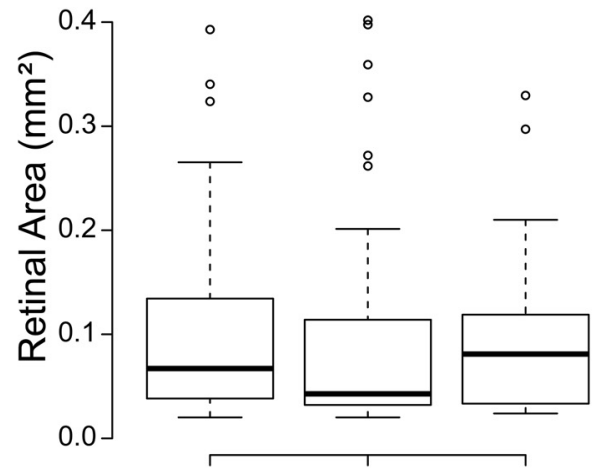

P31 Saline P31 CPPG P31 TGS

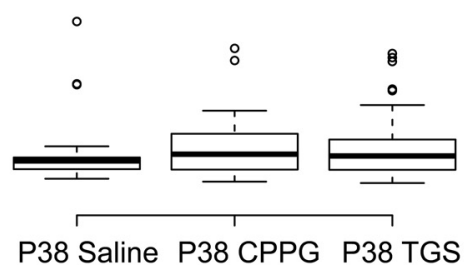

Figure 6. RGC receptive field sizes do not differ after drug treatment. $a, 2 D$ Gaussian fits of representative ganglion cell receptive fields from MEA recordings during a white noise stimulus at P31 (top) and P38 (bottom) after ferrets were intraocularly injected with saline (left), TGS (center), or CPPG (right) from P15-P25. Scale bar, $100 \mu \mathrm{m}\left(\sim 0.9^{\circ}\right.$ visual angle at the ages recorded). $\boldsymbol{b}$, There is no difference in receptive field area across the treatment conditions at either $\mathrm{P} 31$ or P38, but there is a significant decrease in receptive field size between $\mathrm{P} 31$ and $\mathrm{P} 38$ across all conditions.

MEA at eye opening and 1 week after eye opening using the same reverse correlation calculation on the firing responses to the white-noise stimulus used in our in vivo experiments (Fig. 6a). Our MEA recordings were not specific as to which ganglion cells they recorded from and were likely biased as to which classes of ganglion cells were more likely to be detected. We did not find clear clusters in receptive field size that would indicate distinct populations of classes of ganglion cells. Therefore, our recordings likely included heterogeneous ganglion cell populations. Because we did not expect these populations to differ significantly between our experimental groups, all ganglion-cell-receptive fields classified as single units were used in our analysis.

Ganglion-cell-receptive fields were no different among salinetreated $[0.1 \pm 0.01(\mathrm{SEM})$ square millimeters (94 units from 6 retinas)], TGS-treated $[0.09 \pm 0.01$ (SEM) square millimeters (33 units from 3 retinas)], and CPPG-treated $[0.1 \pm 0.01$ (SEM) square millimeters ( 69 units from 4 retinas)] ferrets at either eye opening or 1 week after eye opening [saline, $(0.045 \pm 0.007$ (SEM) square millimeters; 25 units from 2 retinas; TGS, $0.049 \pm$ 0.004 (SEM) square millimeters; 54 units from 4 retinas; and
CPPG, $0.048 \pm 0.004$ (SEM) square millimeters; 61 units from 4 retinas respectively; Fig. 6b], although we did find a significant decrease in ganglion cell receptive field size after the first week following eye opening ( $p<0.0001)$, which is consistent with the refinement of ganglion cell receptive fields after eye opening in the mouse (Koehler et al., 2011). Although we did not measure the development of surrounds in ganglion cells, we observed no difference in center size that might suggest a difference in ganglion cell surrounds that could contribute to the size of receptive fields in the dLGN.

\section{Discussion}

Activity-dependent plasticity of neural circuits has often been explored by altering neuronal firing through exogenous manipulations. Here, we instead investigated whether spontaneous activity manipulations could be used to modify neural circuits during a developmental process. We found that increasing the spike rate, burst rate, and frequency of retinal waves can drive a reduction in the size of geniculate receptive fields during a time when external sources of activity are absent. This reduction in receptive field size is due to a shrinking of receptive field centers, with no impact on the strength of inhibitory surrounds.

The functional maturation of visual receptive fields develops over a period of early visual experience. By increasing the frequency of retinal waves before visual experience, we find a decrease in geniculate receptive field size at eye opening, suggesting that patterned spontaneous retinal activity drives this process before eye opening. However, we do not observe any persistent enhancement in geniculate receptive fields after visual experience begins to play a role in development. It could be that the instructive information of retinal waves is too coarse to refine the system beyond a certain level and the statistical properties of the visual world are more effective at driving further refinement. Perhaps if spontaneous activity were modified to reflect the statistics of the visual world, receptive fields would become even more mature than observed by increasing retinal waves. It does not appear to be the case that the retina is too immature to be refined during the period of retinal waves because it is possible to drive smaller receptive fields before visual experience.

Previous research has shown that convergent inputs from RGCs to the dLGN decrease their number of inputs while increasing the strength of the remaining inputs in the weeks after eye opening (Chen and Regehr, 2000). However, little research has been done on the role that late retinal waves play in directing the development of the retinogeniculate circuit before eye opening. Glutamatergic retinal waves are driven by the extrasynaptic spread of glutamate released by bipolar cells (Firl et al., 2013). In the ferret, they start $\sim \mathrm{P} 10$ and are coincident with cholinergic 
retinal waves driven by starburst amacrine cells (Wong et al., 1993). Between P10 and P20, the spontaneous activity of retinal waves has been shown to be important for the segregation of the C-lamina of the dLGN (Davis et al., 2015) and for the maintenance of the already segregated A-lamina (Chapman, 2000). After P20 in the ferret, the synaptic organization underlying the cholinergic network disassembles and retinal waves are entirely driven by glutamate release (Sun et al., 2008). The retina becomes photoactive shortly thereafter, although the eyes do not open until $\sim$ P30. Our data show for the first time a positive relationship between the level of spontaneous activity during retinal waves and the developmental refinement of the functional properties of geniculate receptive fields before eye opening.

We used two different approaches to increase the spontaneous activity of the retina during glutamatergic retinal waves. The first approach used a combination of strychinine (a glycine antagonist), gabazine (a GABAa antagonist), and TPMPA (a GABAc antagonist, which is particularly robust in the retina) to decrease inhibition. These drugs have been used in other experiments for the purpose of probing the circuitry of the retina (Kerschensteiner and Wong, 2008; Firl et al., 2013) and their effects are well described. They are potent and effective at blocking inhibition and increasing the spontaneous activity of the retina (Firl et al., 2013). The second approach targeted the hyperpolarizing action of the metabatrophic glutamate receptor mGluR6 with the antagonist CPPG. The effect of this drug is to increase the release of glutamate from On bipolar cells, which increases the levels of spontaneous activity and the amount of time that retinal waves are propagating through the retina (Awatramani and Slaughter, 2000).

These alternative approaches toward increasing spontaneous activity differ significantly in the magnitude of the increase in spiking activity, but do not significantly differ in the magnitude of the increase in bursting or waving time (although there is a trend toward greater bursts and waves with inhibition blockade). There also is a trend toward a decrease in the spatiotemporal correlations of spontaneous activity with TGS relative to CPPG treatment, but whether this is meaningful is difficult to determine because both treatments produce the same geniculate receptive field size reduction.

Due to the differences in the magnitude of the effect on spontaneous activity, it is difficult to disentangle the importance of spatiotemporal patterned activity versus overall levels of activity. High-frequency bursts and spatiotemporal correlations, but not individual spike rates, have been shown to be essential components of spontaneous retinal activity in the segregation of eyespecific inputs during stage II retinal waves (Torborg and Feller, 2005) and the timing of bursts rather than the timing of spikes has been suggested to govern the learning rules of plasticity at the retinogeniculate synapse (Butts et al., 2007). Consistent with this notion, our simulation of increased spike rates also indicates that the wave time increase after drug treatment cannot be simply explained by increased spiking activity. Nevertheless, although TGS treatment trends toward more bursts and waves than CPPG treatment, it also trends toward poorer spatiotemporal correlations. These two factors appear to balance out as if by increasing the gain on a nosier signal such that both treatments are equally effective at reducing the size of geniculate receptive fields relative to saline. Although our experiments are limited by the nature of the pharmacology, more targeted approaches differentially adjusting spike and burst rates may be able to identify the elements of patterned activity that govern refinement in the dLGN.

What is the mechanism by which the geniculate receptive fields are smaller at eye opening after increased patterned spon- taneous activity? Our spatial frequency-tuning data suggest that there is a refinement of convergent RGC input driving the center of the receptive field because surround mechanisms are not mature at eye opening in either saline- or drug-treated ferrets. Previous research has argued that the connections from the retina to the dLGN undergo a period of growth, elaboration, and pruning during late retinal waves (Hahm et al., 1999). After eye opening, the maturation of geniculate receptive fields is driven by the clustering of presynaptic boutons and little structural reorganization of ganglion cell arbors (Lin et al., 2014).

There are at least three possible mechanisms by which the receptive fields of geniculate neurons can be refined through spontaneous activity. First, the increase in spontaneous activity can prevent the growth and elaboration of presynaptic arbors, resulting in smaller ganglion cell arbors and less convergence during development. This would result in a smaller sampling of visual space by geniculate neurons and smaller receptive fields. The second possibility is that normal ganglion cell growth and elaboration occurs, but the pruning of overexuberant ganglion cell arbors is accelerated, resulting in smaller geniculate receptive fields earlier. Finally, normal growth, elaboration, and pruning may take place, but the clustering of geniculate synapses onto fewer and more retinotopically aligned ganglion cell receptive fields may be accelerated, resulting in greater synaptic drive from a smaller retinotopic region of visual space. These hypotheses are not mutually exclusive because clustering could also occur in tandem with a failure of elaboration or an acceleration of pruning. However, this clustering might produce faster latencies and our observation of reduced geniculate receptive fields occurred independently of any difference in latency.

Our experiments demonstrate that the developmental refinement of geniculate receptive fields can be accelerated by increasing the levels of patterned spontaneous activity in the retina before eye opening in the ferret, which indicates that spontaneous retinal activity can play an instructive role in this process. This has no consequence on the normal development that occurs after eye opening as a result of normal visual experience, nor does it accelerate the developmental process during this later period. The refinement is likely due to the pruning of convergent inputs from the retina onto geniculate neurons and takes place before the maturation of inhibitory surround mechanisms in the center-surround receptive field structure, which develops later as receptive fields mature under the influence of normal visual experience. Our experiments highlight the capacity of patterned spontaneous activity to instruct the development of neural circuits.

\section{References}

Akerman CJ, Smyth D, Thompson ID (2002) Visual experience before eyeopening and the development of the retinogeniculate pathway. Neuron 36:869-879. CrossRef Medline

Archer SM, Dubin MW, Stark LA (1982) Abnormal development of kitten retino-geniculate connectivity in the absence of action potentials. Science 217:743-745. CrossRef Medline

Awatramani GB, Slaughter MM (2000) Origin of transient and sustained responses in ganglion cells of the retina. J Neurosci 20:7087-7095. Medline

Bansal A, Singer JH, Hwang BJ, Xu W, Beaudet A, Feller MB (2000) Mice lacking specific nicotinic acetylcholine receptor subunits exhibit dramatically altered spontaneous activity patterns and reveal a limited role for retinal waves in forming $\mathrm{ON}$ and OFF circuits in the inner retina. J Neurosci 20:7672-7681. Medline

Baroncelli L, Bonaccorsi J, Milanese M, Bonifacino T, Giribaldi F, Manno I, Cenni MC, Berardi N, Bonanno G, Maffei L, Sale A (2012) Enriched experience and recovery from amblyopia in adult rats: Impact of motor, social and sensory components. Neuropharmacology 62:2388-2397. CrossRef Medline Bisti S, Gargini C, Chalupa LM (1998) Blockade of glutamate-mediated ac- 
tivity in the developing retina perturbs the functional segregation of $\mathrm{ON}$ and OFF pathways. J Neurosci 18:5019-5025. Medline

Blankenship AG, Feller MB (2010) Mechanisms underlying spontaneous patterned activity in developing neural circuits. Nat Rev Neurosci 11:18-29. CrossRef Medline

Burbridge TJ, Xu HP, Ackman JB, Ge X, Zhang Y, Ye MJ, Zhou ZJ, Xu J, Contractor A, Crair MC (2014) Visual circuit development requires patterned activity mediated by retinal acetylcholine receptors. Neuron 84:1049-1064. CrossRef Medline

Butts DA, Rokhsar DS (2001) The information content of spontaneous retinal waves. J Neurosci 21:961-973. Medline

Butts DA, Kanold PO, Shatz CJ (2007) A burst-based "Hebbian" learning rule at retinogeniculate synapses links retinal waves to activity-dependent refinement. PLoS Biol 5:e61. Medline

Cantrell DR, Cang J, Troy JB, Liu X (2010) Non-centered spike-triggered covariance analysis reveals neurotrophin-3 as a developmental regulator of receptive field properties of ON-OFF retinal ganglion cells. PLoS Comput Biol 6:e1000967. CrossRef Medline

Chapman B (2000) Necessity for afferent activity to maintain eye-specific segregation in ferret lateral geniculate nucleus. Science 287:2479-2482. CrossRef Medline

Chapman B, Gödecke I (2000) Cortical cell orientation selectivity fails to develop in the absence of ON-center retinal ganglion cell activity. J Neurosci 20:1922-1930. Medline

Chen C, Regehr WG (2000) Developmental remodeling of the retinogeniculate synapse. Neuron 28:955-966. CrossRef Medline

Cutts CS, Eglen SJ (2014) Detecting pairwise correlations in spike trains: an objective comparison of methods and application to the study of retinal waves. J Neurosci 34:14288-14303. CrossRef Medline

Davis ZW, Sun C, Derieg B, Chapman B, Cheng HJ (2015) Epibatidine blocks eye-specific segregation in ferret dorsal lateral geniculate nucleus during stage III retinal waves. PLoS one 10:e0118783. CrossRef Medline

Enroth-Cugell C, Robson JG (1966) The contrast sensitivity of retinal ganglion cells of the cat. J Physiol 187:517-552. CrossRef Medline

Failor S, Chapman B, Cheng HJ (2015) Retinal waves regulate afferent terminal targeting in the early visual pathway. Proc Natl Acad Sci U S A 112:E2957-2966. CrossRef Medline

Falkenberg T, Mohammed AK, Henriksson B, Persson H, Winblad B, Lindefors N (1992) Increased expression of brain-derived neurotrophic factor mRNA in rat hippocampus is associated with improved spatial memory and enriched environment. Neurosci Lett 138:153-156. CrossRef Medline

Firl A, Sack GS, Newman ZL, Tani H, Feller MB (2013) Extrasynaptic glutamate and inhibitory neurotransmission modulate ganglion cell participation during glutamatergic retinal waves. J Neurophysiol 109:1969-1978. CrossRef Medline

Furman M, Xu HP, Crair MC (2013) Competition driven by retinal waves promotes morphological and functional synaptic development of neurons in the superior colliculus. J Neurophysiol 110:1441-1454. CrossRef Medline

Hahm JO, Cramer KS, Sur M (1999) Pattern formation by retinal afferents in the ferret lateral geniculate nucleus: developmental segregation and the role of N-methyl-D-aspartate receptors. J Comp Neurol 411:327-345. CrossRef Medline

Hooks BM, Chen C (2006) Distinct roles for spontaneous and visual activity in remodeling of the retinogeniculate synapse. Neuron 52:281-291. CrossRef Medline

Huberman AD, Stellwagen D, Chapman B (2002) Decoupling eye-specific segregation from lamination in the lateral geniculate nucleus. J Neurosci 22:9419-9429. Medline

Huberman AD, Murray KD, Warland DK, Feldheim DA, Chapman B (2005) Ephrin-As mediate targeting of eye-specific projections to the lateral geniculate nucleus. Nat Neurosci 8:1013-1021. CrossRef Medline

Huberman AD, Speer CM, Chapman B (2006) Spontaneous retinal activity mediates development of ocular dominance columns and binocular receptive fields in v1. Neuron 52:247-254. CrossRef Medline

Kerschensteiner D, Wong RO (2008) A precisely timed asynchronous pattern of ON and OFF retinal ganglion cell activity during propagation of retinal waves. Neuron 58:851-858. CrossRef Medline

Kerschensteiner D, Morgan JL, Parker ED, Lewis RM, Wong RO (2009) Neurotransmission selectively regulates synapse formation in parallel circuits in vivo. Nature 460:1016-1020. CrossRef Medline

Koehler CL, Akimov NP, Rentería RC (2011) Receptive field center size decreases and firing properties mature in ON and OFF retinal ganglion cells after eye opening in the mouse. J Neurophysiol 106:895-904. CrossRef Medline

Lin DJ, Kang E, Chen C (2014) Changes in input strength and number are driven by distinct mechanisms at the retinogeniculate synapse. J Neurophysiol 112:942-950. CrossRef Medline

Liu N, He S, Yu X (2012) Early natural stimulation through environmental enrichment accelerates neuronal development in the mouse dentate gyrus. PLoS One 7:e30803. CrossRef Medline

Meister M, Wong RO, Baylor DA, Shatz CJ (1991) Synchronous bursts of action potentials in ganglion cells of the developing mammalian retina. Science 252:939-943. CrossRef Medline

Movshon JA, Kiorpes L, Hawken MJ, Cavanaugh JR (2005) Functional maturation of the macaque's lateral geniculate nucleus. J Neurosci 25:27122722. CrossRef Medline

Penn AA, Riquelme PA, Feller MB, Shatz CJ (1998) Competition in retinogeniculate patterning driven by spontaneous activity. Science 279:2108 2112. CrossRef Medline

Pfeiffenberger C, Cutforth T, Woods G, Yamada J, Rentería RC, Copenhagen DR, Flanagan JG, Feldheim DA (2005) Ephrin-As and neural activity are required for eye-specific patterning during retinogeniculate mapping. Nat Neurosci 8:1022-1027. CrossRef Medline

Reid RC, Victor JD, Shapley RM (1997) The use of m-sequences in the analysis of visual neurons: linear receptive field properties. Vis Neurosci 14:1015-1027. CrossRef Medline

Schwartz N, Schohl A, Ruthazer ES (2011) Activity-dependent transcription of BDNF enhances visual acuity during development. Neuron 70 : 455-467. CrossRef Medline

Shoham S, Fellows MR, Normann RA (2003) Robust, automatic spike sorting using mixtures of multivariate t-distributions. J Neurosci Methods 127:111-122. CrossRef Medline

Speer CM, Mikula S, Huberman AD, Chapman B (2010) The developmental remodeling of eye-specific afferents in the ferret dorsal lateral geniculate nucleus. Anat Rec (Hoboken) 293:1-24. CrossRef Medline

Stafford BK, Sher A, Litke AM, Feldheim DA (2009) Spatial-temporal patterns of retinal waves underlying activity-dependent refinement of retinofugal projections. Neuron 64:200-212. CrossRef Medline

Stellwagen D, Shatz CJ (2002) An instructive role for retinal waves in the development of retinogeniculate connectivity. Neuron 33:357-367. CrossRef Medline

Stryker MP, Harris WA (1986) Binocular impulse blockade prevents the formation of ocular dominance columns in cat visual cortex. J Neurosci 6:2117-2133. Medline

Sun C, Speer CM, Wang GY, Chapman B, Chalupa LM (2008) Epibatidine application in vitro blocks retinal waves without silencing all retinal ganglion cell action potentials in developing retina of the mouse and ferret. J Neurophysiol 100:3253-3263. CrossRef Medline

Tavazoie SF, Reid RC (2000) Diverse receptive fields in the lateral geniculate nucleus during thalamocortical development. Nat Neurosci 3:608-616. CrossRef Medline

Tian N, Copenhagen DR (2003) Visual stimulation is required for refinement of ON and OFF pathways in postnatal retina. Neuron 39:85-96. CrossRef Medline

Torborg CL, Feller MB (2005) Spontaneous patterned retinal activity and the refinement of retinal projections. Prog Neurobiol 76:213-235. CrossRef Medline

Warland DK, Huberman AD, Chalupa LM (2006) Dynamics of spontaneous activity in the fetal macaque retina during development of retinogeniculate pathways. J Neurosci 26:5190-5197. CrossRef Medline

Wong RO, Meister M, Shatz CJ (1993) Transient period of correlated bursting activity during development of the mammalian retina. Neuron 11: 923-938. CrossRef Medline

Xu HP, Furman M, Mineur YS, Chen H, King SL, Zenisek D, Zhou ZJ, Butts DA, Tian N, Picciotto MR, Crair MC (2011) An instructive role for patterned spontaneous retinal activity in mouse visual map development. Neuron 70:1115-1127. CrossRef Medline

Yu K, Wu Y, Zhang Q, Xie H, Liu G, Guo Z, Li F, Jia J, Kuang S, Hu R (2014) Enriched environment induces angiogenesis and improves neural function outcomes in rat stroke model. J Neurol Sci 347:275-280. CrossRef Medline

Zahs KR, Stryker MP (1985) The projection of the visual field onto the lateral geniculate nucleus of the ferret. J Comp Neurol 241:210-224. CrossRef Medline 